\title{
Human acetyl-CoA carboxylase 2 expressed in silkworm Bombyx mori exhibits posttranslational biotinylation and phosphorylation
}

\author{
In-Wook Hwang • Yu Makishima • Tatsuya Kato • \\ Sungjo Park • Andre Terzic • Enoch Y. Park
}

Received: 18 February 2014 / Accepted: 23 March 2014 / Published online: 17 April 2014

(C) The Author(s) 2014. This article is published with open access at Springerlink.com

\begin{abstract}
Biotin-dependent human acetyl-CoA carboxylases (ACCs) are integral in homeostatic lipid metabolism. By securing posttranslational biotinylation, ACCs perform coordinated catalytic functions allosterically regulated by phosphorylation/dephosphorylation and citrate. The production of authentic recombinant ACCs is heeded to provide a reliable tool for molecular studies and drug discovery. Here, we examined whether the human ACC2 (hACC2), an isoform of ACC produced using the silkworm BmNPV bacmid system, is equipped with proper posttranslational modifications to carry out catalytic functions as the silkworm harbors an inherent posttranslational modification machinery. Purified hACC2 possessed genuine biotinylation capacity probed by
\end{abstract}

I.-W. Hwang • E. Y. Park

Laboratory of Biotechnology, Integrated Bioscience Section, Graduate School of Science and Technology, Shizuoka University, 836 Ohya, Suruga-ku, Shizuoka 422-8529, Japan

Y. Makishima $\cdot$ T. Kato $\cdot$ E. Y. Park

Laboratory of Biotechnology, Department of Applied Biological Chemistry, Faculty of Agriculture, Shizuoka University, 836 Ohya, Suruga-ku, Shizuoka 422-8529, Japan

T. Kato $・$ E. Y. Park $(\bowtie)$

Laboratory of Biotechnology, Green Chemistry Research Division, Research Institute of Green Science and Technology, Shizuoka University, 836 Ohya Suruga-ku, Shizuoka 422-8529, Japan

e-mail: acypark@ipc.shizuoka.ac.jp

S. Park $\cdot$ A. Terzic

Center for Regenerative Medicine, Mayo Clinic, 200 First Street SW, Rochester, MN 55905, USA

S. Park $\cdot$ A. Terzic $(\bowtie)$

Marriott Heart Disease Research Program, Division of

Cardiovascular Diseases, Departments of Medicine, Molecular

Pharmacology and Experimental Therapeutics, and Medical

Genetics, Mayo Clinic, 200 First Street SW, Rochester, MN 55905,

USA

e-mail: terzic.andre@mayo.edu biotin-specific streptavidin and biotin antibodies. In addition, phosphorylated hACC2 displayed limited catalytic activity whereas dephosphorylated hACC2 revealed an enhanced enzymatic activity. Moreover, hACC2 polymerization, analyzed by native page gel analysis and atomic force microscopy imaging, was allosterically regulated by citrate and the phosphorylation/dephosphorylation modulated citrateinduced hACC2 polymerization process. Thus, the silkworm BmNPV bacmid system provides a reliable eukaryotic protein production platform for structural and functional analysis and therapeutic drug discovery applications implementing suitable posttranslational biotinylation and phosphorylation.

Keywords Human acetyl-CoA carboxylase 2 (hACC2) . Phosphorylation/dephosphorylation · Lipid metabolism . Silkworm · Bombyx mori nucleopolyhedrovirus

\section{Introduction}

Acetyl-CoA carboxylases (ACCs) are biotin-dependent enzymes catalyzing the production of malonyl-CoA from acetyl-CoA, a critical metabolic intermediate in lipid metabolism (Brownsey et al. 2006; Kim 1997; Saggerson 2008; Tong 2013; Wakil and Abu-Elheiga 2009). Two different isoforms of ACC, ACC1 and ACC2, partake in lipid metabolism in humans and mammals (Abu-Elheiga et al. 1995, 1997; Ha et al. 1996). ACC1, encoded by $A C A C A$, predominantly exists in the cytosol of lipogenic organs such as adipose tissue and liver where malonyl-CoA functions as a substrate for long chain fatty acids synthesis. In contrast, $A C A C B$-encoded $\mathrm{ACC} 2$ is associated with the outer membrane of mitochondria in oxidative tissues such as the heart, liver, and skeletal muscle where malonyl-CoA is utilized as a negative regulator of fatty acid oxidation. Due to the bifunctional roles in catabolic and anabolic metabolism, ACC functions as a bioenergetics 
controller to promote stem cell function and tissue regeneration to regulate lipid homeostasis (Folmes et al. 2013; Fullerton et al. 2013; Knobloch et al. 2013; Park et al. 2013). Moreover, ACC2 knockout demonstrates anti-obesity effects and prevention of cardiac remodeling (Abu-Elheiga et al. 2001, 2003; Kolwicz et al. 2012). Therefore, ACC activity regulation has been recognized as an attractive therapeutic target for dysregulated lipid metabolism such as obesity, diabetes, cancer, and cardiovascular disease (Tong and Harwood 2006).

Eukaryotic ACCs, unlike prokaryotic ACCs composed of three separate functional proteins, comprises three distinctive functional domains, including a biotin carboxylase (BC) domain, a biotin carboxyl carrier protein domain (BCCP), and a carboxyltransferase (CT) domain, to carry out multiple functions. Integral in catalysis is biotin, a prosthetic group attached to lysine residue within the BCCP domain (Bianchi et al. 1990; Cronan and Waldrop 2002; Tanabe et al. 1975; Tong 2013). The BC domain catalyzes the Mg-ATP-dependent carboxylation of biotin to form carboxybiotin using bicarbonate as the $\mathrm{CO}_{2}$ donor (reaction 1). Then, carboxybiotin is transferred to the CT domain mediating transfer of the carboxyl group from carboxybiotin to acetyl-CoA to form malonyl-CoA (reaction 2). Besides these core catalytic reactions, ACC activities are allosterically regulated by multiple factors including phosphorylation/dephosphorylation and citrate (Beaty and Lane 1983a; Brownsey et al. 2006; Ha et al. 1994; Meredith and Lane 1978; Munday et al. 1988; Wojtaszewski et al. 2003). Thus, ACC with posttranslational biotinylation and properly regulated by allosteric modulators is essential in evaluating the full functionality of multistep reactions to unfold its functional mechanisms and systematic inhibitor discovery efforts.

$$
\begin{aligned}
\mathrm{ATP}-\mathrm{Mg}_{2}{ }^{+} & +\mathrm{HCO}_{3}^{-}+\mathrm{ACC}-\text { biotin } \leftrightarrow \mathrm{ACC}-\text { biotin- } \mathrm{CO}_{2}^{-} \\
& +\mathrm{ADP}+\mathrm{P}_{\mathrm{i}}
\end{aligned}
$$

$$
\begin{aligned}
& \text { ACC-biotin- } \mathrm{CO}_{2}^{-}+\text {acetyl-CoA } \leftrightarrow \text { malonyl-CoA } \\
& \quad+\text { ACC-biotin }
\end{aligned}
$$

The baculovirus expression system has been considered as the most efficient eukaryotic heterologous protein expression system as the host insect cells can implement foolproof posttranslational modifications similar to higher eukaryotes (Kost et al. 2005; Possee 1997). Two types of baculovirus expression systems, i.e., Autographa california multiple nucleopolyhedrovirus (AcMNPV) and Bombyx mori nucleopolyhedrovirus (BmNPV) systems, have been widely used (Kost et al. 2005; Maeda 1989). We developed a BmNPV bacmid, an Escherichia coli and B. mori hybrid shuttle vector, to expedite the heterologous protein production platform without construction and amplification of viruses in $B$. mori culture cells as recombinant BmNPV DNA can be directly injected into silkworm pupae or larvae (Hiyoshi et al. 2007; Motohashi et al. 2005; Park et al. 2008a). Using the BmNPV bacmid system, intracellular, extracellular, and membrane proteins have been successfully generated with proper folding and posttranslational modifications (Kato et al. 2010, 2012; Otsuki et al. 2013).

Here, we examined whether the recombinant human $\mathrm{ACC} 2$, produced using the silkworm BmNPV bacmid-based approach, secures proper posttranslational modifications to fulfill the essential catalysis and allosteric modulation. We report that $\mathrm{ACC} 2$, demonstrating consistent catalytic activities with proper posttranslational biotinylation and phosphorylation, is regulated by allosteric modulation. Thus, silkwormbased BmNPV system provides a reliable large-scale protein production platform for structural and function studies as well as drug discovery applications implementing essential posttranslational modifications.

\section{Materials and methods}

\section{Construction of recombinant hACC2 BmNPV bacmid}

The human ACC2 (2,548 amino acids) is associated with the mitochondria membrane through $\mathrm{N}$-terminal 148 hydrophobic amino acids classified as mitochondrial attachment and target sequences (Tong 2013). To increase the solubility of recombinant human ACC2 (hACC2), these hydrophobic residues were excluded using polymerase chain reaction (PCR) with the following set of primers, 5'-GCCGTCGACATGTCCA AAGAAGACAAGAAGCAG-3' (forward), 5'-GCTCTAGA TTACTTGTCATCGTCATCCTTGTAGTCGGTGGAGGC CGGGCTGTCCATG-3' (reverse), and designated as $\triangle 148$ aa-hACC2. The PCR cycle was performed following 30 cycles of denaturation at $94^{\circ} \mathrm{C}$ for $30 \mathrm{~s}$, annealing at $55^{\circ} \mathrm{C}$ for $30 \mathrm{~s}$, and extension at $72^{\circ} \mathrm{C}$ for $7 \mathrm{~min}$, followed by a final extension at $72{ }^{\circ} \mathrm{C}$ for $10 \mathrm{~min}$. The complementary DNA of human ACC2 from Mammalian Gene Collection (Thermo Scientific, Pittsburgh, PA, USA) was used as a template. The resultant PCR product was digested with $S a l \mathrm{I}$ and $X b a \mathrm{I}$ followed by purification with a GFX ${ }^{\mathrm{TM}}$ PCR DNA and Gel Band Purification Kit (GE Healthcare, Amersham, UK). The purified DNA fragment was ligated into pFastbac 1 vector, which was transformed into $E$. coli competent $\mathrm{DH} 5 \alpha$ cells (Invitrogen, Carlsbad, CA, USA) and cultured on a solid LB medium containing $100 \mu \mathrm{g} / \mathrm{mL}$ of ampicillin at $37^{\circ} \mathrm{C}$ for $18 \mathrm{~h}$ to generate recombinant plasmid. The plasmid containing human $\triangle 148$ aa-hACC2 gene was isolated and identified by DNA sequencing. Finally, E. coli $\mathrm{BmDH} 10 \mathrm{bac}-\mathrm{CP}^{-}-\mathrm{Chi}$ competent cells containing the cysteine proteinase- and chitinase-deficient BmNPV bacmid (Park et al. 2008a) were transformed with the pFastbac1- $\Delta 148 \mathrm{aa}-\mathrm{hACC} 2$ and cultured on a solid LB medium containing $50 \mu \mathrm{g} / \mathrm{mL}$ of kanamycin, 
$7 \mu \mathrm{g} / \mathrm{mL}$ of gentamycin, $10 \mu \mathrm{g} / \mathrm{mL}$ of tetracycline, $40 \mu \mathrm{g} / \mathrm{mL}$ of isopropyl $\beta$-D-1-thiogalactopyranoside (IPTG), and $100 \mu \mathrm{g} / \mathrm{mL}$ of 5-bromo-4-chloro-3-indolyl-4-galactoside (XGal) (Takara Bio Inc., Otsu Shiga, Japan) at $37{ }^{\circ} \mathrm{C}$ for $18 \mathrm{~h}$. The bacmid containing BmNPV- $\triangle 148$ aa-hACC2 was isolated from white positive colonies.

Expression and purification of recombinant hACC2 in silkworm

Silkworm pupae were used for the expression of recombinant $\triangle 148$ aa-hACC2 as a bioreactor. To produce recombinant protein in pupae, $10 \mu \mathrm{g}$ of BmNPV- $\Delta 148 \mathrm{aa}-\mathrm{hACC} 2$ bacmid DNA was directly injected with DMRIE-C reagent (Invitrogen) into the dorsal of pupae. The injected pupae were reared at $27^{\circ} \mathrm{C}$ for $6-7$ days, and stored at $-80{ }^{\circ} \mathrm{C}$ until further analysis. Protein purification was carried out at $4{ }^{\circ} \mathrm{C}$ to minimize aggregation and protease activity. Five pupae were homogenized in $10 \mathrm{~mL}$ of lysis buffer $(50 \mathrm{mM}$ Tris- $\mathrm{HCl}$, $150 \mathrm{mM} \mathrm{NaCl}, \mathrm{pH} \mathrm{7.4}$, and $0.1 \%$ TritonX-100) containing an EDTA-free protease inhibitor tablet (Roche, Mannheim, Germany) using a homogenizer (GLH-115, Yamato, Tokyo, Japan). Cell debris was removed by pelleting through centrifugation at $12,000 \times \mathrm{g}$ for $30 \mathrm{~min}$. The supernatant was filtered using a $0.45-\mu \mathrm{m}$ syringe filter and loaded onto a $500-\mu \mathrm{L}$ of Anti-FLAG M2 antibody Affinity Gel (Sigma-Aldrich, St. Louis, MO, USA) pre-equilibrated with equilibration buffer (50 mM Tris-HCl, $150 \mathrm{mM} \mathrm{NaCl}, \mathrm{pH} 7.4$ and $0.02 \%$ TritonX-100). The column was washed with $2.5 \mathrm{~mL}$ of equilibration buffer and eluted with elution buffer $(100 \mu \mathrm{g} / \mathrm{mL}$ FLAG peptide in $50 \mathrm{mM}$ Tris- $\mathrm{HCl}$ and $150 \mathrm{mM} \mathrm{NaCl}$, $\mathrm{pH} 7.4)$. The eluted $\triangle 148 \mathrm{aa}-\mathrm{hACC} 2$ was collected and concentrated using a $100 \mathrm{~K}$ Amicon Ultra centrifugal filter (Millipore, Billerica, MA, USA).

Confirmation of biotinylation and phosphorylation by Western blotting

The posttranslational biotinylation and phosphorylation of purified $\Delta 148$ aa-hACC2 were measured by Western blotting analysis. Prior to electrophoresis, purified sample was boiled for $5 \mathrm{~min}$ at $95{ }^{\circ} \mathrm{C}$ with protein denaturing buffer (Nacalai Tesque, Kyoto, Japan). Samples were electrophoresed in a $5 \%$ SDS-PAGE gel with the Mini-protean system (Bio-Rad, Hercules, CA, USA) at $150 \mathrm{~V}$ for 45-60 min in Tris-glycine buffer $(25 \mathrm{mM}$ Tris, $250 \mathrm{mM}$ glycine, $\mathrm{pH} 8.3$, and $0.1 \%$ SDS). The separated proteins on a SDS-PAGE gel were transferred to PVDF membranes (GE Healthcare) by electroblotting on a wet blotter (Bio-Rad) at $15 \mathrm{~V}$ for $1 \mathrm{~h}$. To detect the purified $\triangle 148 \mathrm{aa}-\mathrm{hACC} 2$ and their biotinylation and phosphorylation, several specific antibodies were used. A mouse anti-FLAG antibody (Wako Pure Chem. Ind. Ltd., Osaka, Japan) was used to detect purified $\triangle 148$ aa-hACC2 as a primary antibody. A monoclonal anti-phosphoserine antibody (Sigma-Aldrich) was used for phosphorylation detection as a primary antibody. An anti-mouse IgG-HRP (GE Healthcare) was used for above both cases as a secondary antibody. A goat anti-biotin antibody (Abcam, Cambridge, MA, USA) and streptavidin HRP conjugate (Thermo Scientific, Rockford, IL, USA) were used for biotinylation detection as a primary antibody. A rabbit anti-goat IgG-HRP (Santa Cruz Biotechnology, Santa Cruz, CA, USA) and an anti-mouse IgG-HRP were used as a secondary antibody.

\section{Dephosphorylation of $\Delta 148$ aa-hACC2}

Dephosphorylation was carried out using Lambda protein phosphatase (Lambda PP; New England Biolabs, Ipswich, MA, USA). The purified $\triangle 148 \mathrm{aa}-\mathrm{hACC} 2$ was incubated with $0.5 \mu \mathrm{L}$ of Lambda PP, $1 \mathrm{X}$ NEBuffer for protein metallophosphatases (PMP) and $1 \mathrm{mM} \mathrm{MnCl}_{2}$ at $30{ }^{\circ} \mathrm{C}$ for $0,1,3,6 \mathrm{~h}$. Sterilized water instead of Lambda PP was used as negative control. Dephosphorylation was confirmed by Western blot using a monoclonal anti-phosphoserine antibody (Sigma-Aldrich) produced in mouse and an anti-mouse IgGHRP (GE Healthcare). The activity of dephosphorylated $\triangle 148$ aa-hACC2 was determined using ACC assay.

\section{Acetyl-CoA carboxylase assay}

To measure ACC activity, $4 \mu \mathrm{L}$ of purified $\Delta 148 \mathrm{aa}-\mathrm{hACC} 2$ was incubated with $36 \mu \mathrm{L}$ of reaction buffer $(50 \mathrm{mM}$ of HEPES, $\mathrm{pH} 7.4,5 \mathrm{mM}$ of $\mathrm{NaHCO}_{3}, 10 \mathrm{mM}$ of $\mathrm{MgCl}_{2}$, $10 \mathrm{mM}$ of sodium citrate, $0.5 \%$ of DMSO, $4 \mathrm{mM}$ of ATP, and $0.4 \mathrm{mM}$ of acetyl-CoA) at $37{ }^{\circ} \mathrm{C}$. The reaction was terminated by addition of $4 \mu \mathrm{L}$ of $100 \%$ trichloroacetic acid. The produced phosphate during the reaction was determined using a SensoLyte ${ }^{\circledR}$ MG Phosphate Assay Kit (AnaSpec, Fremont, CA, USA) by measuring the absorbance at $655 \mathrm{~nm}$. Protein concentration was determined using BCA protein assay kit (Thermo Scientific).

\section{Citrate-induced polymerization of $\Delta 148 \mathrm{aa}-\mathrm{hACC} 2$}

In order to confirm the allosteric regulation of purified $\triangle 148$ aa-hACC2 from silkworm, citrate-induced polymerization was evaluated. The purified $\triangle 148$ aa-hACC2 was incubated with $50 \mathrm{mM}$ of HEPES (pH 7.4), $1 \mathrm{mM}$ of dithiothreitol (DTT), and different concentration of citrate at $37{ }^{\circ} \mathrm{C}$ for $30 \mathrm{~min}$. The polymerization was evaluated by Native-PAGE and Western blotting. Purified proteins were prepared in a non-denaturing sample buffer (Native sample buffer, BioRad). Samples were electrophoresed in a $5 \%$ Native-PAGE (without SDS) with the Mini-protean system (Bio-Rad) at $150 \mathrm{~V}$ for 45-60 min in Tris-glycine buffer (25 mM Tris and $250 \mathrm{mM}$ glycine, $\mathrm{pH}$ 8.3). Next, Western blotting protocol 
was used as described above. The antibodies for detecting polymerized $\Delta 148$ aa-hACC2 were same as used for detecting purified $\triangle 148$ aa-hACC2. The activity of $\triangle 148$ aa-hACC2 by citrate concentration-dependent polymerization was determined by ACC assay.

\section{Atomic force microscopy}

Nanoscale AFM imaging was employed to investigate the dynamic forms polymers due to allosteric regulation of hACC2 2 by citrate. The $\triangle 148$ aa-hACC2 was incubated with or without $15 \mathrm{mM}$ citrate for $20 \mathrm{~min}$ at $37^{\circ} \mathrm{C}$. The resultant mixtures were placed on the freshly cleaved mica surface and incubated for several hours in a moisture chamber. After washing with water and drying under nitrogen, the samples were subjected to tapping mode AFM imaging on the Nanoscope IV PicoForce Multimode AFM, equipped with an E-scanner and a rectangular-shaped silicon cantilever (Bruker, Madison, WI, USA) with a 42-N/m spring constant and a resonant frequency of $\sim 300 \mathrm{kHz}$ at ambient environment (Park et al. 2008b; Park and Terzic 2010). Images (512× 512 pixels/image) were collected from each sample with maximum image size of $5 \times 5 \mu \mathrm{m}$ and analyzed using the Nanoscope Version 6.13 software (Bruker).

\section{Results}

Expression and purification of recombinant $\Delta 148$ aa-hACC2

Human ACC2 is a large polypeptide comprised of a mitochondrial attachment domain, a mitochondrial target sequence domain, a biotin carboxylase domain, a biotin carboxyl carrier protein domain, and a carboxyltransferase domain (Fig. 1a) (Bianchi et al. 1990; Tanabe et al. 1975; Tong 2013). Biotin is covalently attached to lysine within BCCP domain through posttranslational modification, and several serine residues are phosphorylated by protein kinases (Beaty and Lane 1983a; Brownsey et al. 2006; Ha et al. 1994; Meredith and Lane 1978). We deleted the N-terminal 148 hydrophobic amino acids to enhance the solubility of heterologous protein, which retains core functional modules (Fig. 1b). In particular, to prevent protease activity and liquefaction of heterologous proteins in silkworm-based expression system, E. coli BmDH10bac- $C P^{-}-C h i^{-}$competent cells were employed.

Recombinant $\triangle 148$ aa-hACC2 with a C-terminal FLAG tag was purified using an anti-FLAG M2 affinity gel column. Eluted with FLAG peptides, the enriched protein migrated to $\sim 260 \mathrm{kDa}$, a predicted molecular weight, on SDS-PAGE based on comparison with molecular weight markers (Fig. 2a). Western blot analysis using a FLAG-specific antibody confirmed the expression of hACC2 (Fig. 2b). In addition, the yield of final purified $\Delta 148$ aa-hACC2 was $495 \mu \mathrm{g} /$ pupa. This pupae-based recombinant protein expression provided a high yield of purified $\Delta 148$ aa-hACC2 compared to expression in silkworm larvae $(150 \mu \mathrm{g} /$ larva) (Park et al. 2013). The purified hACC2 displayed significant homogeneity on SDS-PAGE and Western blot analysis, thereby further functional analysis was carried out using this enriched $\triangle 148$ aa-hACC2.

\section{Biotinylation of $\Delta 148 \mathrm{aa}-\mathrm{hACC} 2$}

The posttranslational modification with biotin in ACC2 is essential to implement catalytic function. The biotin binding residue in human ACC2 has not been clearly identified, yet structural studies using nuclear magnetic resonance suggests that biotin is attached to lysine 929 within a BCCP domain (Lee et al. 2008). The biotinylation of $\triangle 148$ aa-hACC2 from the silkworm was analyzed by Western blotting using an antibiotin antibody. To further validate biotinylation of the $\triangle 148$ aa-hACC2, streptavidin HRP conjugate was employed to detect the biotin group as streptavidin is known to interact with biotin with very high affinity. Although the hACC2-anti-

\section{A: Native human ACC2}

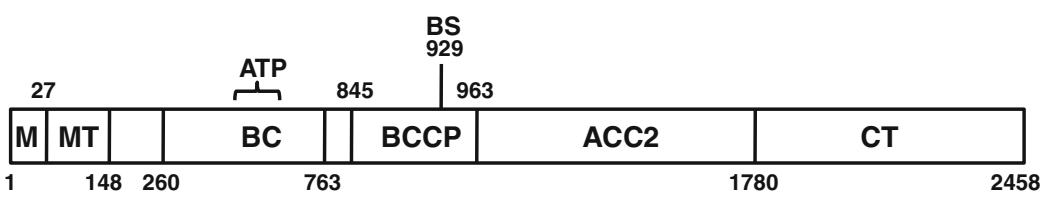

\section{B: Recombinant $\triangle 148$ aa-human ACC2}

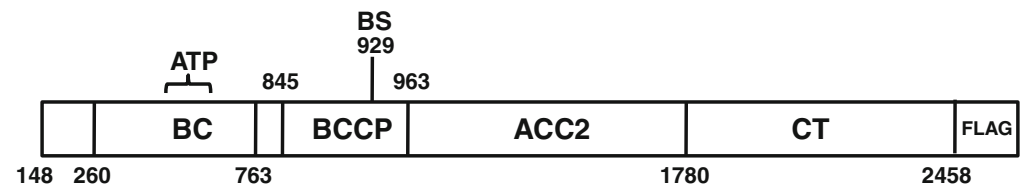

Fig. 1 A schematic presentation of human ACC2 domains. a Native hACC2. ACC2, human acetyl-CoA carboxylase 2; ATP, ATP-grasp domain; $B C$, biotin carboxylase domain; $B C C P$, biotin carboxyl carrier protein domain; $B S$, biotinylation site; $C T$, carboxyltransferase domain;
$M$, membrane attachment domain; $M T$, mitochondria targeting sequence. (b) Recombinant $\triangle 148$ aa-hACC2. N-terminal 148 amino acids were deleted for increasing the solubility. FLAG was tagged at its C-terminus for affinity purification 


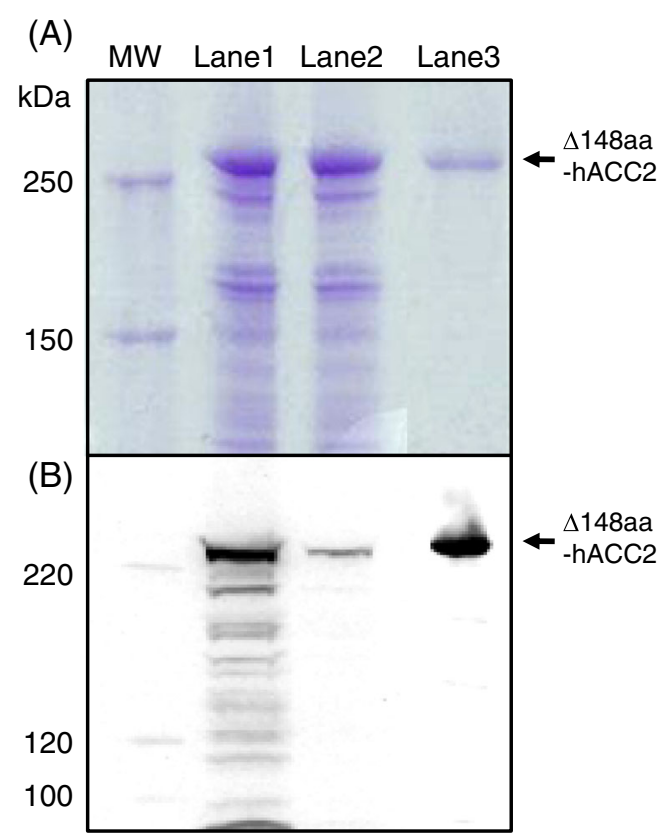

Fig. 2 The expression of recombinant $\triangle 148$ aa-hACC2 was confirmed by analysis of SDS-PAGE (a) and Western blot (b). $M W$, molecular weight markers; Lane 1, protein extracts after infection; Lane 2, flow through during FLAG-tag purification; Lane 3, purified and concentrated $\triangle 148$ aa-hACC2. An anti-FLAG M2 antibody and an anti-mouse IgGHRP were used to detect purified $\triangle 148$ aa-hACC2

biotin band was detected more intensely than the streptavidin bound band (Fig. 3), biotin-specific detection using two different methods confirmed hACC2 biotinylation. Collectively, without additional supplement of biotin to generate biotinylated ACC observed in Trichoplusia ni cells (Kim et al. 2007), silkworm enables to produce biotinylated heterologous proteins.

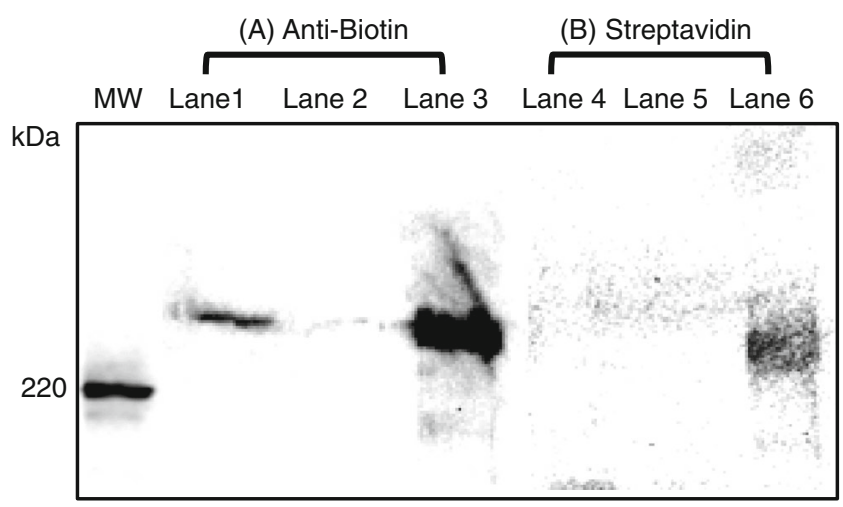

Fig. 3 The purified $\triangle 148$ aa-hACC2 possesses posttranslational biotinylation confirmed by Western blot analysis using an anti-biotin antibody $(A)$ and streptavidin HRP conjugate $(B) . M W$, molecular weight markers; Lanes 1 and 4, protein extracts after infection; Lanes 2 and 5 , flow through during FLAG-tag purification; Lanes 3 and 6, purified and concentrated $\triangle 148$ aa-hACC2. An anti-biotin antibody and a streptavidin HRP conjugate were used as primary antibodies. A rabbit anti-goat IgG$\mathrm{HRP}$ and an anti-mouse IgG-HRP were used as secondary antibodies
Phosphorylation and dephosphorylation of $\Delta 148$ aa-hACC2

Adenosine monophosphate-activated protein kinase (AMPK)-mediated phosphorylation is other layer of posttranslational modification to allosterically regulate ACC catalytic function. Phosphorylation inactivates ACC catalytic activity whereas dephosphorylation activates the enzymatic function. Notably, phosphorylation of Ser222 in hACC2 (Ser212 in mouse ACC2) has been recognized as a vital process for homeostatic lipid metabolism (Fullerton et al. 2013; Wakil and Abu-Elheiga 2009). Consistent with these findings, the crystal structure of biotin carboxylase domain of hACC2 has revealed that the phosphorylation of Ser222 disrupts the polymerization of $\mathrm{ACC} 2$, a widely recognized mechanism in modulating catalytic function (Cho et al. 2010; Lee et al. 2008).

We evaluated posttranslational phosphorylation of recombinant $\triangle 148$ aa-hACC2, and then whether the phosphorylated protein could be effectively dephosphorylated accompanying the changes of catalytic function. Western blotting analysis using a monoclonal anti-phosphoserine antibody demonstrated the phosphorylation of $\triangle 148$ aa-hACC2 purified from silkworm pupae (Fig. 4a). The addition of Lambda protein phosphatase, a $\mathrm{Mn}^{2+}$-dependent dephosphorylation enzyme, gradually decreased the phosphorylation compared with control (Fig. 4a), yet dephosphorylation was not completely achieved in $\triangle 148$ aa-hACC2 up to $6 \mathrm{~h}$ incubation. This finding suggests that some of phosphorylation sites in full-length hACC2 could be readily inaccessible by Lambda protein phosphatase unlike isolated functional domains such as a biotin carboxylase domain (Kwon et al. 2013).

The effect of dephosphorylation was assessed by measuring the catalytic function of $\triangle 148$ aa-hACC2 (Fig. 4b). $\triangle 148$ aa-hACC2 was treated with and without Lambda protein phosphatase for $2 \mathrm{~h}$, and then catalytic activity was measured as a function of incubation time. Purified $\Delta 148$ aahACC2 with indigenous posttranslational phosphorylation provided a specific activity of $0.786 \pm 0.229 \mathrm{nmol} \mathrm{P}_{\mathrm{i}} / \mathrm{mg} / \mathrm{min}$ $(n=6)$, whereas phosphatase treated $\Delta 148 \mathrm{aa}-\mathrm{hACC} 2$ protein yielded a specific activity of $1.336 \pm 0.441 \mathrm{nmol} \mathrm{P}_{\mathrm{i}} / \mathrm{mg} / \mathrm{min}$ $(n=6)$, about 2-fold increase. These measurements are consistent with the findings observed in knock-in mice samples where Ser212 (mouse sequence) is replaced with alanine to ablate the critical serine phosphorylation (Fullerton et al. 2013).

\section{Allosteric activation of $\Delta 148 \mathrm{aa}-\mathrm{hACC} 2$ by citrate}

Citrate-induced polymerization has been extensively employed to understand the regulatory mechanism of ACC, although the concentrations of citrate required for allosteric activation are much higher than that present at physiological locale (Beaty and Lane 1983a, b; Gregolin et al. 1966; Kim 

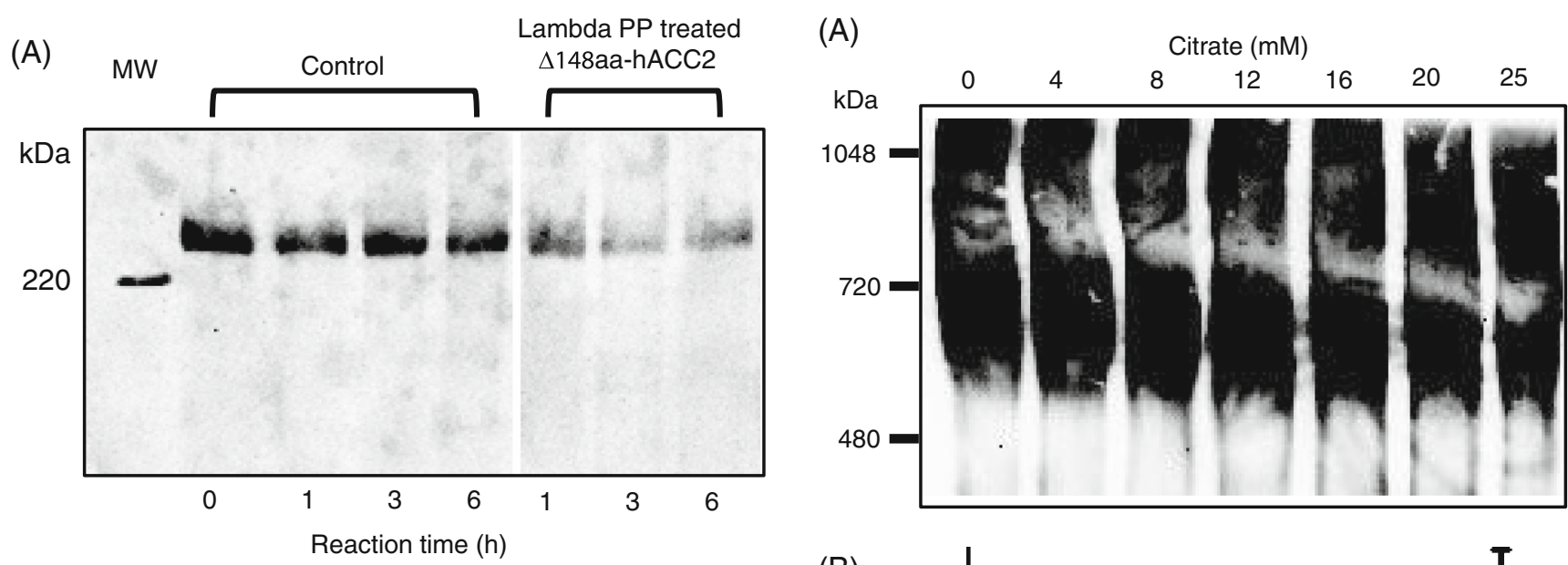

(B)

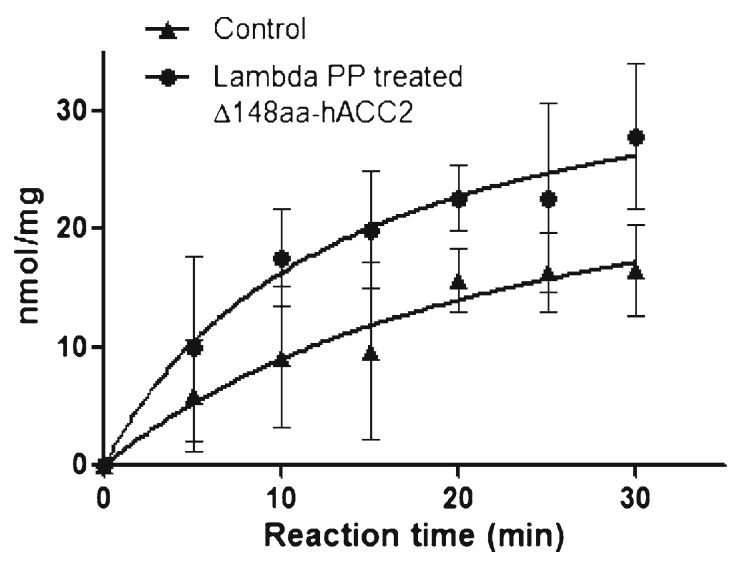

(B)

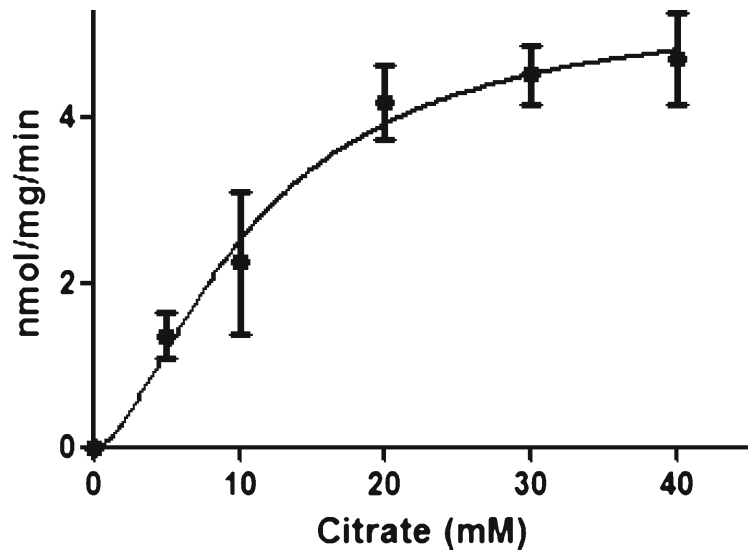

Fig. 5 Polymerization and enzyme activities of $\triangle 148$ aa-hACC2 were modulated by citrate concentration. a Degree of polymerization of $\triangle 148$ aa-hACC2 by different concentration of citrate $(0,4,8,12,16$, 20 , and $25 \mathrm{mM}$ ). The polymerization was confirmed using Native-PAGE. b Enzymatic activities by citrate concentration. All data are means \pm S.D. from three separate experiments

et al. 2010). Upon incubation with citrate, ACC polymerizes into filamentous structures containing 10-20 protomer units with increased functional activity (Kim et al. 2007; Locke et al. 2008). Regardless of biological significance of citrate in ACC regulation, the citrate binding sites have not been identified.

The citrate-induced allosteric activation of $\Delta 148$ aahACC2 was investigated by measuring the modulation of structural and functional properties. Incubation of $\Delta 148 \mathrm{aa}-$ hACC2 with citrate generated the formation of high molecular weight polymers detected on Native-PAGE, which was increased with rising citrate concentrations (Fig. 5a). This polymerization results indicate that $\triangle 148$ aa-hACC2 derived from pupae consists of dimers and tetramers based on comparison with molecular weight markers, and increased citrate concentrations led to tetramer production by decreasing dimers (Fig. 5a). Consistent with dosedependent polymerization, the catalytic activity of $\triangle 148$ aa-hACC2 was also enhanced with increasing citrate concentrations (Fig. 5b). Incubation of $\triangle 148$ aa-hACC2

with 5,10 , and $20 \mathrm{mM}$ citrate produced specific activity of $1.363 \pm 0.279,2.246 \pm 0.870$, and $4.186 \pm 0.200 \mathrm{nmol} \mathrm{P}_{\mathrm{i}} /$ $\mathrm{mg} / \mathrm{min}(n=4)$, respectively. These values indicated that when the citrate concentration was increased by 2 -fold, activities were also increased by about 2-fold in proportion to citrate concentration (Fig. 5b). Furthermore, when the concentration of citrate exceeds $20 \mathrm{mM}$, the activation curve follows a sigmoidal response, consistent with previous findings (Cheng et al. 2007).

The structural changes of $\triangle 148 \mathrm{aa}-\mathrm{hACC} 2$ by citrate were also evaluated using high-resolution AFM at nanoscale resolution (Fig. 6). The purified $\triangle 148$ aa-hACC2 without citrate showed almost homogeneous particle distribution. However, citrate addition to $\Delta 148 \mathrm{aa}-$ hACC2 generated filamentous polymeric forms, significantly larger than $\triangle 148 \mathrm{aa}-\mathrm{hACC} 2$ alone. These findings not only support the formation of high molecular weight polymers observed in Native-PAGE but also validate that $\triangle 148$ aa-hACC2 produced in silkworm possess full functionality with proper allosteric modulations. 
(A) $\triangle 148 \mathrm{aa}-\mathrm{hACC} 2$

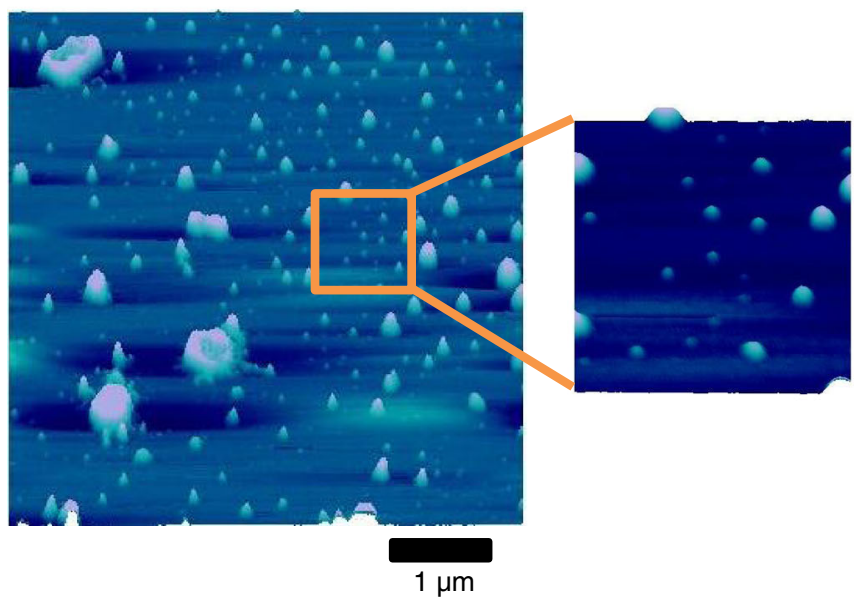

(B) $\triangle 148 \mathrm{aa}-\mathrm{hACC} 2+15 \mathrm{mM}$ citrate

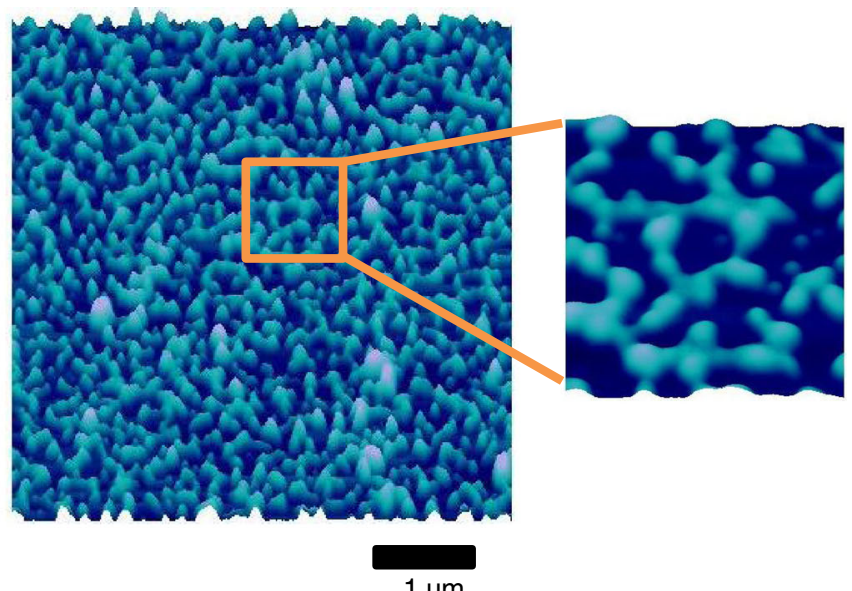

$1 \mu \mathrm{m}$

Fig. 6 Atomic force microscopy nanoscale images of $\Delta 148$ aa-hACC2. a $\Delta 148$ aa-hACC2 alone. b $\Delta 148$ aa-hACC2 with $15 \mathrm{mM}$ citrate after 20 -min incubation at $37^{\circ} \mathrm{C}$. Citrate induced the filaments formation

\section{Discussion}

Acetyl-CoA carboxylase is a multidomain and multifunctional protein working as an energetic controller in homeostatic lipid metabolism participating in fatty acid synthesis and fatty acid oxidation (Tong 2013; Wakil and Abu-Elheiga 2009). The catalytic function of ACC through a biotin prosthetic group can be allosterically regulated by multiple factors including posttranslational phosphorylation and dephosphorylation (Wakil and Abu-Elheiga 2009). In addition, tertiary level regulation of ACC with small acidic proteins, i.e., Spot14 and Mig12, has been recently identified (Colbert et al. 2010; Kim et al. 2010; Knobloch et al. 2013; Park et al. 2013). Particularly, due to the pathophysiological relevance of ACC2 in lipid metabolic syndrome associated with obesity, diabetes, cancer, and cardiovascular disease, ACC2 activity regulation has been considered as a candidate target for therapeutic interventions, which essentially requires authentic bioengineered recombinant proteins (Abu-Elheiga et al. 2001; Tong 2013; Tong and Harwood 2006). Here, we successfully produced with high fidelity human ACC2 using the silkworm BmNPV system armed with a proper posttranslational modification machinery. The heterologous ACC2 harbors all necessary posttranslational biotinylation and phosphorylation, vital to maintain functional integrity. Thus, the silkworm BmNPV bacmid system provides a reliable largescale production platform for eukaryotic proteins required for posttranslational modifications.

Biotin is a water-soluble vitamin serving as a vital prosthetic group involved in five carboxylases in human (Zempleni et al. 2009). In hACC2, biotin is covalently linked to lysine 929 within a BCCP domain and forms carboxybiotin using bicarbonate as the $\mathrm{CO}_{2}$ donor. Following a large conformational change, the carboxyl group from carboxybiotin is transferred to acetyl-CoA to produce malonyl-CoA (Tong
2013). We revealed posttranslational biotinylation of functional recombinant hACC2 from silkworm, without any additional supplement of biotin under silkworm rearing conditions. We believe that this is the first demonstration of posttranslational biotinylation in proteins expressed in silkworm B. mori.

ACC phosphorylation/dephosphorylation is one of the allosteric regulatory mechanisms. Phosphorylation of ACC by AMP-activated protein kinase and cAMP-dependent protein kinase inhibits the enzymatic activity of ACC, whereas dephosphorylation activates the catalytic function (Munday et al. 1988). Although several phosphorylation sites have been identified, Ser212 (mouse sequence) phosphorylation was recently recognized as an integral process in activity modulation (Fullerton et al. 2013) where knock-in mice with substitution of Ser212 with alanine displayed an increased ACC2 activity. Consistent with this, we demonstrated about a 2-fold increased specific activity in dephosphorylated hACC2 compared to the phosphorylated counterpart, underscoring that the critical serine residue is fully accessible to phosphatase protein. ACC is also allosterically activated by citrate, which is a metabolic intermediate produced in mitochondrial tricarboxylic acid cycle. Although the concentrations of citrate required to increase the enzymatic activity of ACC are much higher than the physiological concentrations of citrate, citrate has been widely used to modulate ACC function (Beaty and Lane 1983a; Cheng et al. 2007; Thampy and Wakil 1988). We demonstrated herein citrate-induced hACC2 catalytic function enhancement and filamentous polymer formation. In summary, a multifunctional human ACC2 was successfully produced using silkworm-based protein expression system. Heterologous ACC2 was correctly folded with posttranslational biotinylation and phosphorylation, retaining catalytic activity and citrate-induced allosteric regulation. Moreover, silkworm demonstrated a high yield of recombinant ACC2 production. Thus, the silkworm-based BmNPV expression 
method equipped with a proper posttranslational modification machinery provides a large-scale eukaryotic protein production platform for structural and functional research particularly in the application of therapeutic drug discovery.

Acknowledgments This work was supported partly by the Strategic Young Researcher Overseas Visits Program for Accelerating Brain Circulation from Japan Society for the Promotion of Science (JSPS), Japan, the National Institutes of Health, and Marriott Heart Disease Research Program, Mayo Clinic. A.T. holds the Marriott Family Professorship in Cardiovascular Diseases Research.

Open Access This article is distributed under the terms of the Creative Commons Attribution License which permits any use, distribution, and reproduction in any medium, provided the original author(s) and the source are credited.

\section{References}

Abu-Elheiga L, Jayakumar A, Baldini A, Chirala SS, Wakil SJ (1995) Human acetyl-CoA carboxylase: characterization, molecular cloning, and evidence for two isoforms. Proc Natl Acad Sci U S A 92: 4011-4015

Abu-Elheiga L, Almarza-Ortega DB, Baldini A, Wakil SJ (1997) Human acetyl-CoA carboxylase 2. Molecular cloning, characterization, chromosomal mapping, and evidence for two isoforms. J Biol Chem 272:10669-10677

Abu-Elheiga L, Matzuk MM, Abo-Hashema KA, Wakil SJ (2001) Continuous fatty acid oxidation and reduced fat storage in mice lacking acetyl-CoA carboxylase 2. Science 291:2613-2616. doi: 10.1126/science. 1056843

Abu-Elheiga L, Oh W, Kordari P, Wakil SJ (2003) Acetyl-CoA carboxylase 2 mutant mice are protected against obesity and diabetes induced by high-fat/high-carbohydrate diets. Proc Natl Acad Sci U S A 100:10207-10212. doi:10.1073/pnas.1733877100

Beaty NB, Lane MD (1983a) Kinetics of activation of acetyl-CoA carboxylase by citrate. Relationship to the rate of polymerization of the enzyme. J Biol Chem 258:13043-13050

Beaty NB, Lane MD (1983b) The polymerization of acetyl-CoA carboxylase. J Biol Chem 258:13051-13055

Bianchi A, Evans JL, Iverson AJ, Nordlund AC, Watts TD, Witters LA (1990) Identification of an isozymic form of acetyl-CoA carboxylase. J Biol Chem 265:1502-1509

Brownsey RW, Boone AN, Elliott JE, Kulpa JE, Lee WM (2006) Regulation of acetyl-CoA carboxylase. Biochem Soc Trans 34: 223-227. doi:10.1042/BST20060223

Cheng D, Chu CH, Chen L, Feder JN, Mintier GA, Wu Y, Cook JW, Harpel MR, Locke GA, An Y, Tamura JK (2007) Expression, purification, and characterization of human and rat acetyl coenzyme A carboxylase (ACC) isozymes. Protein Expr Purif 51:11-21. doi: 10.1016/j.pep.2006.06.005

Cho YS, Lee JI, Shin D, Kim HT, Jung HY, Lee TG, Kang LW, Ahn YJ, Cho HS, Heo YS (2010) Molecular mechanism for the regulation of human ACC2 through phosphorylation by AMPK. Biochem Biophys Res Commun 391:187-192. doi:10.1016/j.bbrc.2009.11. 029

Colbert CL, Kim CW, Moon YA, Henry L, Palnitkar M, McKean WB, Fitzgerald K, Deisenhofer J, Horton JD, Kwon HJ (2010) Crystal structure of Spot 14, a modulator of fatty acid synthesis. Proc Natl Acad Sci U S A 107:18820-18825. doi:10.1073/pnas.1012736107

Cronan JE, Waldrop GL (2002) Multi-subunit acetyl-CoA carboxylases. Prog Lipid Res 41:407-435
Folmes CD, Park S, Terzic A (2013) Lipid metabolism greases the stem cell engine. Cell Metab 17:153-155. doi:10.1016/j.cmet.2013.01.010

Fullerton MD, Galic S, Marcinko K, Sikkema S, Pulinilkunnil T, Chen ZP, O'Neill HM, Ford RJ, Palanivel R, O'Brien M, Hardie DG, Macaulay SL, Schertzer JD, Dyck JRB, van Denderen BJ, Kemp BE, Steinberg GR (2013) Single phosphorylation sites in Acc1 and Acc2 regulate lipid homeostasis and the insulin-sensitizing effects of metformin. Nat Med 19:1649-1654. doi:10.1038/nm.3372

Gregolin C, Ryder E, Kleinschmidt AK, Warner RC, Lane MD (1966) Molecular characteristics of liver acetyl CoA carboxylase. Proc Natl Acad Sci U S A 56:148-155

Ha J, Daniel S, Broyles SS, Kim KH (1994) Critical phosphorylation sites for acetyl-CoA carboxylase activity. J Biol Chem 269:22162-22168

Ha J, Lee JK, Kim KS, Witters LA, Kim KH (1996) Cloning of human acetyl-CoA carboxylase-beta and its unique features. Proc Natl Acad Sci U S A 93:11466-11470

Hiyoshi M, Kageshima A, Kato T, Park EY (2007) Construction of a cysteine protease deficient Bombyx mori multiple nucleopolyhedrovirus bacmid and its application to improve expression of a fusion protein. J Virol Methods 144:91-97. doi:10.1016/j. jviromet.2007.04.005

Kato T, Kajikawa M, Maenaka K, Park EY (2010) Silkworm expression system as a platform technology in life science. Appl Microbiol Biotechnol 85:459-470. doi:10.1007/s00253-009-2267-2

Kato T, Suzuki F, Park EY (2012) Display of the human (pro)renin receptor on Bombyx mori nucleopolyhedrovirus (BmNPV) particles using Bm cells. J Biosci Bioeng 114:564-569. doi:10.1016/j.jbiosc. 2012.06.008

Kim KH (1997) Regulation of mammalian acetyl-coenzyme A carboxylase. Annu Rev Nutr 17:77-99. doi:10.1146/annurev.nutr.17.1.77

Kim KW, Yamane H, Zondlo J, Busby J, Wang M (2007) Expression, purification, and characterization of human acetyl-CoA carboxylase 2. Protein Expr Purif 53:16-23. doi:10.1016/j.pep.2006.11.021

Kim CW, Moon YA, Park SW, Cheng D, Kwon HJ, Horton JD (2010) Induced polymerization of mammalian acetyl-CoA carboxylase by MIG12 provides a tertiary level of regulation of fatty acid synthesis. Proc Natl Acad Sci U S A 107:9626-9631. doi:10.1073/pnas. 1001292107

Knobloch M, Braun SMG, Zurkirchen L, von Schoultz C, Zamboni N, Araúzo-Bravo MJ, Kovacs WJ, Karalay O, Suter U, Machado RAC, Roccio M, Lutolf MP, Semenkovich CF, Jessberger S (2013) Metabolic control of adult neural stem cell activity by Fasndependent lipogenesis. Nature 493:226-230. doi:10.1038/ nature 11689

Kolwicz SC, Olson DP, Marney LC, Garcia-Menendez L, Synovec RE, Tian R (2012) Cardiac-specific deletion of acetyl CoA carboxylase 2 prevents metabolic remodeling during pressure-overload hypertrophy. Circ Res 111:728-738. doi:10.1161/CIRCRESAHA.112. 268128

Kost TA, Condreay JP, Jarvis DL (2005) Baculovirus as versatile vectors for protein expression in insect and mammalian cells. Nat Biotechnol 23:567-575. doi:10.1038/nbt1095

Kwon S, Cho Y, Heo Y (2013) Structural insights into the regulation of ACC2 by citrate. Bull Korean Chem Soc 34:565-568. doi:10.5012/ bkcs.2013.34.2.565

Lee CK, Cheong HK, Ryu KS, Lee JI, Lee W, Jeon YH, Cheong C (2008) Biotinoyl domain of human acetyl-CoA carboxylase: structural insights into the carboxyl transfer mechanism. Proteins 72:613624. doi:10.6564/JKMRS.2008.12.1.001

Locke GA, Cheng D, Witmer MR, Tamura JK, Haque T, Carney RF, Rendina AR, Marcinkeviciene J (2008) Differential activation of recombinant human acetyl-CoA carboxylases 1 and 2 by citrate. Arch Biochem Biophys 475:72-79. doi:10.1016/j.abb.2008.04.011

Maeda S (1989) Expression of foreign genes in insects using baculovirus vectors. Annu Rev Entomol 34:351-372. doi:10.1146/annurev.en. 34.010189.002031 
Meredith MJ, Lane MD (1978) Acetyl-CoA carboxylase. Evidence for polymeric filament to protomer transition in the intact avian liver cell. J Biol Chem 253:3381-3383

Motohashi T, Shimojima T, Fukagawa T, Maenaka K, Park EY (2005) Efficient large-scale protein production of larvae and pupae of silkworm by Bombyx mori nuclear polyhedrosis virus bacmid system. Biochem Biophys Res Commun 326:564-569. doi:10.1016/j. bbrc.2004.11.060

Munday MR, Campbell DG, Carling D, Hardie DG (1988) Identification by amino acid sequencing of three major regulatory phosphorylation sites on rat acetyl-CoA carboxylase. Eur J Biochem 175:331-338

Otsuki T, Dong J, Kato T, Park EY (2013) Expression, purification and antigenicity of Neospora caninum-antigens using silkworm larvae targeting for subunit vaccines. Vet Parasitol 192:284-287. doi:10. 1016/j.vetpar.2012.09.038

Park S, Terzic A (2010) Quaternary structure of KATP channel SUR2A nucleotide binding domains resolved by synchrotron radiation $\mathrm{X}$-ray scattering. J Struct Biol 169:243-251

Park EY, Abe T, Kato T (2008a) Improved expression of fusion protein using a cysteine- protease- and chitinase-deficient Bombyx mori (silkworm) multiple nucleopolyhedrovirus bacmid in silkworm larvae. Biotechnol Appl Biochem 49:135-140. doi:10.1042/BA20070098

Park S, Lim BB, Perez-Terzic C, Mer G, Terzic A (2008b) Interaction of asymmetric $\mathrm{ABCC} 9$-encoded nucleotide binding domains determines KATP channel SUR2A catalytic activity. J Proteome Res 7: 1721-1728. doi:10.1021/pr7007847

Park S, Hwang IW, Makishima Y, Perales-Clemente E, Kato T, Niederländer NJ, Park EY, Terzic A (2013) Spot14/Mig12 heterocomplex sequesters polymerization and restrains catalytic function of human acetyl-CoA carboxylase 2. J Mol Recognit 26: 679-688. doi:10.1002/jmr.2313

Possee RD (1997) Baculoviruses as expression vectors. Curr Opin Biotechnol 8:569-572. doi:10.1016/S0958-1669(97)80030-4

Saggerson D (2008) Malonyl-CoA, a key signaling molecule in mammalian cells. Annu Rev Nutr 28:253-272. doi:10.1146/annurev.nutr. 28.061807.155434

Tanabe T, Wada K, Okazaki T, Numa S (1975) Acetyl-coenzyme-A carboxylase from rat liver. Subunit structure and proteolytic modification. Eur J Biochem 57:15-24

Thampy KG, Wakil SJ (1988) Regulation of acetyl-coenzyme A carboxylase I. Purification and properties of two forms of acetyl-coenzyme A carboxylase from rat liver. J Biol Chem 263:6447-6453

Tong L (2013) Structure and function of biotin-dependent carboxylases. Cell Mol Life Sci 70:863-891. doi:10.1007/s00018-012-1096-0

Tong L, Harwood HJ (2006) Acetyl-coenzyme A carboxylases: versatile targets for drug discovery. J Cell Biochem 99:1476-1488. doi:10. $1002 / j \mathrm{cb} .21077$

Wakil SJ, Abu-Elheiga LA (2009) Fatty acid metabolism: target for metabolic syndrome. J Lipid Res 50(Suppl):S138-S143

Wojtaszewski JF, MacDonald C, Nielsen JN, Hellsten Y, Hardie DG, Kemp BE, Kiens B, Richter EA (2003) Regulation of 5'AMPactivated protein kinase activity and substrate utilization in exercising human skeletal muscle. Am J Physiol Endocrinol Metab 284: E813-E822. doi:10.1152/ajpendo.00436.2002

Zempleni J, Wijeratne SSK, Hassan YI (2009) Biotin. Biofactors 35:3646. doi:10.1002/biof.8 